\title{
Factors Influencing Timing Resolution in a Commercial LSO PET Camera
}

\author{
W. W. Moses, Senior Member, IEEE, and M. Ullisch
}

\begin{abstract}
The CPS Accel is a commercial PET camera based on a block detector with 64 LSO scintillator crystals (each $6.75 \mathrm{x}$ $6.75 \times 25 \mathrm{~mm}$ ) read out with 4 photomultiplier tubes. The excellent timing resolution of LSO suggests that this camera might be used for time-of-flight (TOF) PET, thereby reducing the statistical noise significantly. Although the Accel achieves 3 ns coincidence resolution (a factor of two better than BGObased PET cameras), its timing resolution is nearly an order of magnitude worse than that demonstrated with individual LSO crystals. This paper quantifies the effect on the timing of each component in the Accel timing chain to identify which components most limit the camera's timing resolution. The components in the timing chain are: the scintillator crystal, the photomultiplier tube (PMT), the constant fraction discriminator (CFD), and the time to digital converter (TDC). To measure the contribution of each component, we construct a single crystal test system with high-performance versions of these components. This system achieves 221 ps fwhm coincidence timing resolution, which is used as a baseline measurement. One of the high-performance components is replaced by a production component, the coincidence timing resolution is remeasured, and the difference between measurements is the contribution of that (production) component. We find that the contributions of the TDC, CFD, PMT, and scintillator are 2000 ps, $1354 \mathrm{ps,} 422 \mathrm{ps}$, and $326 \mathrm{ps}$ fwhm respectively, and that the overall timing resolution scales like the square root of the amount of scintillation light detected by the PMT. Based on these measurements we predict that the limit for the coincidence timing resolution in a practical, commercial, LSO-based PET camera is $\mathbf{5 2 8}$ ps fwhm.
\end{abstract}

\section{INTRODUCTION}

$T^{\mathrm{T}}$ has long been recognized that by accurately measuring 1 the difference in arrival times of the $511 \mathrm{keV}$ photons coming from positron annihilation, the statistical noise in PET (positron emission tomography) can be reduced. The variance reduction is estimated by $2 \mathrm{D} / c \Delta \mathrm{t}$, where $\mathrm{D}$ is the diameter of the object being imaged, $c$ is the speed of light, and $\Delta \mathrm{t}$ is the coincidence timing measurement accuracy. A number of PET cameras incorporating time-of-flight (TOF) measurement were built in the 1980's [1-8]. These cameras achieved timing resolution of $\sim 500 \mathrm{ps}$ and observed the

Manuscript received Nov 14, 2004. This work was supported in part by the U.S. Department of Energy under contract Nos. DE-AC03-76SF00098 and DE-AC02-05CH11231, and in part by Public Health Service Grant No. R33-EB001928.

W. W. Moses is with Lawrence Berkeley National Laboratory, Berkeley, CA 94720 USA (telephone: ++1-510-486-4432, e-mail: wwmoses@lbl.gov).

M. Ullisch is with University of Hamburg, Hamburg, Germany (e-mail: marcus@ullisch.de). anticipated improvement in statistical noise. Achieving this timing resolution required $\mathrm{BaF}_{2}$ or $\mathrm{CsF}$ scintillators, which imposed tradeoffs that degraded other PET performance aspects. The disadvantages seemed to outweigh the advantages, as few TOF PET cameras were constructed after the 1980's [9] and research on time-of-flight PET essentially halted shortly thereafter. In recent years, several new scintillators have been developed [10-15], expanding the number of choices available to tomograph manufacturers and increasing interest in TOF PET [16-21].

One particularly promising new scintillator is LSO $\left(\mathrm{Lu}_{2} \mathrm{SiO}_{5}: \mathrm{Ce}\right)[22,23]$. Its relatively high light output $(25,000$ to 30,000 photons / MeV) and short scintillation decay time (38 ns), suggest that it should have excellent timing resolution, and timing resolutions under $300 \mathrm{ps}$ fwhm have been measured under ideal conditions [16, 24]. While this resolution was achieved using crystals whose geometry was optimized for timing, resolution slightly better than $500 \mathrm{ps}$ fwhm has been achieved with $3 \times 3 \times 30 \mathrm{~mm}^{3}$ crystal geometries suitable for PET [16]. Several commercial PET cameras have been constructed with LSO (the HRRT [25-27] and the Accel), but they have achieved only 2-3 ns fwhm timing resolution [28]. While this is a factor of 2 better than that typically achieved with BGO scintillator, it is significantly worse than what has been measured with LSO under ideal conditions. The purpose of this paper is to identify the factors that limit the timing performance of existing commercial LSO PET cameras and to predict the achievable timing resolution.

\section{MATERIALS AND METHODS}

To measure the timing contributions in a commercial PET camera, we assume that the relevant components are shown

a)

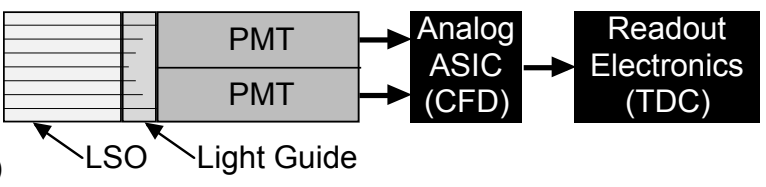

b)

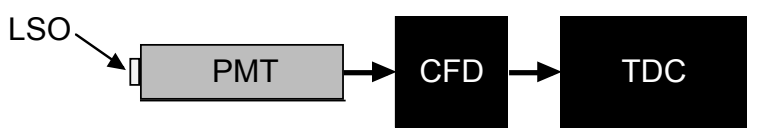

Figure 1: Components of the timing chain. The contributions to timing resolution are from the scintillator crystal (the material, the geometry, the location of the crystal within the detector module, and scatter within the light guide), the PMTs (transit time jitter and propagation time differences), the CFD, and the TDC. The components used in a "commercial" system (a) are slightly different than those used in a "high-performance" system (b). 


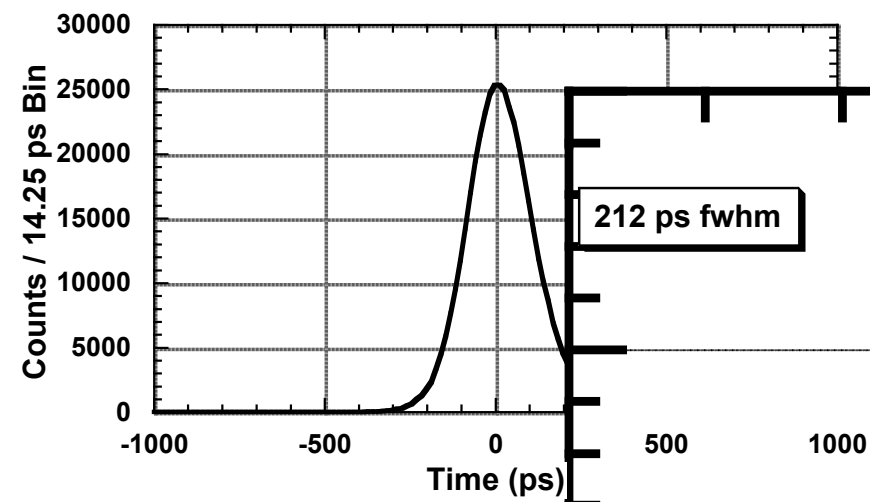

Figure 2: Coincidence timing resolution of a pair of the $\mathrm{BaF}_{2}$-based detector modules that are used to generate the timing trigger.

in Figure 1a. These are a scintillator crystal, a photomultiplier tube (PMT), a constant fraction discriminator (CFD), and a time to digital converter (TDC). We construct a test system that replaces all of the "commercial" versions of the components shown in Figure 1a with "high-performance" versions of these components, as shown in Figure 1b. The coincidence timing resolution of the test system is measured and is considered to be the baseline measurement. One of the four "high-performance" components (scintillator, PMT, CFD, or TDC) is replaced with a "production" version of that component, the coincidence timing resolution remeasured, and the difference (in quadrature) is considered the "extra" timing error of that "production" component.

\section{A. Trigger Signal}

In order to measure timing resolution, a reference trigger signal must be generated. A pair of reference detectors are constructed, each consisting of a $10 \mathrm{~mm}$ cube of $\mathrm{BaF}_{2}$ scintillator coupled to a Hamamatsu H-5321 PMT assembly operated at $-2300 \mathrm{~V}$ and read out with one channel of a Canberra 454 NIM CFD. The pair is excited with coincident $511 \mathrm{keV}$ annihilation photons from a $120 \mu \mathrm{Ci}{ }^{68} \mathrm{Ge}$ source, and the time difference of each event measured with an Ortec 556 NIM TAC and a National Instruments PXI-7831R 16-bit ADC read out by a personal computer. This measurement, shown in Figure 2, yields 212 ps fwhm coincidence timing resolution, implying a trigger accuracy of $150 \mathrm{ps}$ fwhm. The electronics accuracy is estimated by triggering both CFDs with the same input signal, yielding a timing resolution of 25 ps fwhm (<2 ADC bins).

One $\mathrm{BaF}_{2}$ module and the $120 \mu \mathrm{Ci}{ }^{68} \mathrm{Ge}$ source are used to generate reference triggers for all of the subsequent measurements presented in this work. For this and for all subsequent measurements, we do not do "energy gating," that is, we do not integrate the output signal and accept only events above a fixed energy threshold. Instead, the only threshold is the voltage threshold in the CFD. While this threshold provides some coarse energy selection, the threshold is set low $(<50 \mathrm{keV})$ for all measurements.

\section{B. High-Performance Components}

For "high-performance" components, we use a $3 \times 8 \times 12 \mathrm{~mm}^{3}$ piece of LSO scintillator wrapped with Teflon

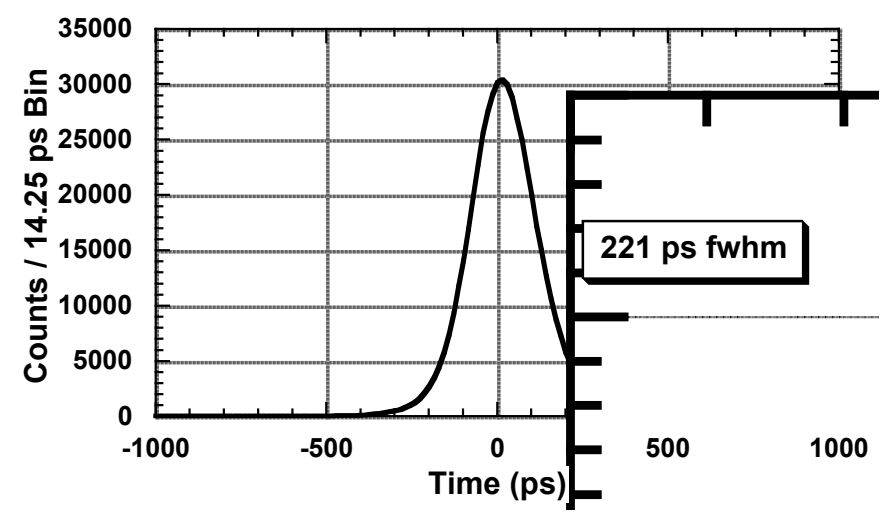

Figure 3: Coincidence timing resolution of the "high-performance" system.

tape reflector on 5 sides and coupled on the $8 \times 12 \mathrm{~mm}^{2}$ side, a Hamamatsu H-5321 PMT assembly operated at $-2300 \mathrm{~V}$, a Canberra 454 NIM CFD, an Ortec 556 NIM TAC, and a National Instruments PXI-7831R 16-bit ADC read out by a personal computer. Except for the scintillator crystal, the components are identical to those used to generate the reference trigger. The coincidence timing resolution measured with this system, shown in Figure 3, is $221 \mathrm{ps}$ fwhm, which is used as the baseline measurement. This "baseline" contains the contributions of the reference trigger uncertainty, as well as the timing uncertainty in the "highperformance" detector module and electronics. Subtracting (in quadrature) the $150 \mathrm{ps}$ fwhm contribution from the trigger yields a measurement of 162 ps fwhm for this LSO crystal / PMT / NIM electronics combination, which is only slightly

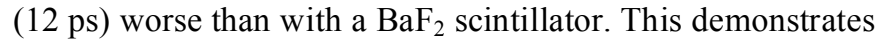
that LSO is capable of outstanding timing resolution.

\section{Production Components}

The commercial camera explored is the initial version of the CPS Accel. Its geometry is very similar to that of the ECAT EXACT, except that it uses LSO rather than BGO scintillator. Detector modules are slightly larger than $50 \mathrm{~mm}$ square and consist of an $8 \times 8$ array of $6.75 \times 6.75 \times 25 \mathrm{~mm}^{3}$ LSO scintillator crystals read out with 4 PMTs. This camera is presently available with two enhancements - a "Hi-Rez" version (with a module that is still $\sim 50 \mathrm{~mm}$ square but subdivides the scintillator block into a $13 \times 13$ array of smaller LSO crystals) and a "Pico-Timing" version (that uses upgraded electronics). The components used here are from the original version of the Accel and do not contain the enhanced "High-Rez" or "Pico-Timing" components.

\section{MEASUREMENTS}

\section{A. Scintillator Crystal}

Twelve individual $6.75 \times 6.75 \times 25 \mathrm{~mm}^{3}$ LSO scintillator crystals were obtained from CPS Innovations. The crystals are the same size, have the same surface finish, and are surrounded (on 5 sides) with the same reflector material as the individual crystals used in the Accel detector modules. A crystal was coupled with MeltMount coupling compound $(n=1.582)$ to a Hamamatsu H-5321 PMT assembly operated at $-2300 \mathrm{~V}$ and read out with one channel of a Canberra 454 


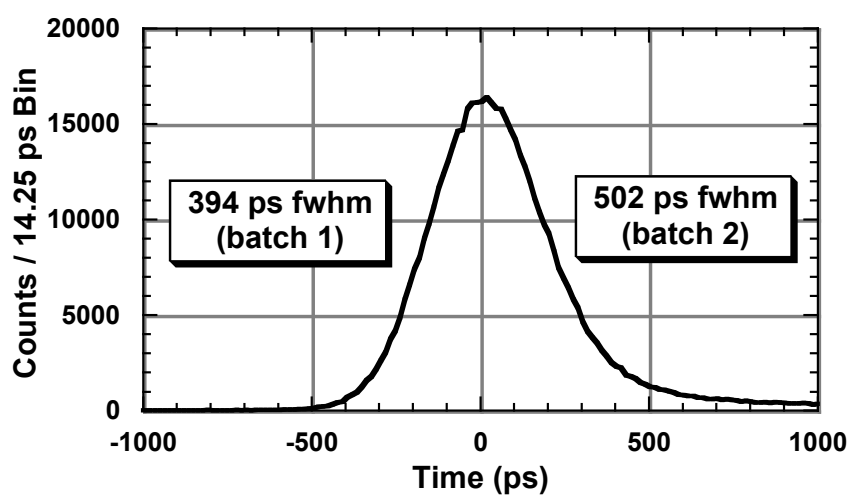

Figure 4: Coincidence timing resolution of a representative "batch 1" Accel crystal attached to the "high-performance" system.

CFD, an Ortec 556 TAC, and a National Instruments PXI7831R 16-bit ADC. One $\mathrm{BaF}_{2}$ module and the $120 \mu \mathrm{Ci}{ }^{68} \mathrm{Ge}$ source are used to generate reference triggers. In other words, it is identical to the "high-performance" setup, but with the "high-performance" $3 \times 8 \times 12 \mathrm{~mm}^{3}$ piece of LSO scintillator replaced by an Accel crystal.

For each crystal, a coincidence timing distribution such as the one shown in Figure 4 is acquired and the width of the distribution is computed. In order to reduce the effects of statistical variations, this measurement is repeated with the remaining 11 crystals and the average timing resolution (i.e., the width of the timing distribution averaged over the crystals) computed. The crystals arrived from CPS in two batches of six crystals each. As we observe statistically significant differences between the two batches, but none within each batch, we present data from each batch separately. The results are shown in Table 1 and indicate that the average timing resolution is $394 \mathrm{ps}$ and $502 \mathrm{ps}$ fwhm for batch 1 and batch 2 respectively. The root mean square

Table 1: Coincidence timing resolution of each type of component in the Accel PET camera. Error bars are the RMS component-to-component variation in the measured timing resolution, and the number of each type of component is given in the last column.

\begin{tabular}{|l|c|c|c|}
\hline Component & $\begin{array}{c}\text { Measured } \\
\text { Resolution } \\
\text { (ps fwhm) }\end{array}$ & $\begin{array}{c}\text { "Extra" } \\
\text { Factor } \\
\text { (ps fwhm) }\end{array}$ & $\begin{array}{c}\text { Number of } \\
\text { Components }\end{array}$ \\
\hline $\begin{array}{l}\text { Scintillator } \\
\text { (batch 1) }\end{array}$ & $394 \pm 13$ & 326 & 6 \\
\hline $\begin{array}{l}\text { Scintillator } \\
\text { (batch 2) }\end{array}$ & $502 \pm 17$ & 451 & 8 \\
\hline $\begin{array}{l}\text { PMT } \\
\text { (Photonis) }\end{array}$ & $530 \pm 51$ & 482 & 8 \\
\hline $\begin{array}{l}\text { PMT } \\
\text { (Hamamatsu) }\end{array}$ & $1372 \pm 193$ & 1354 & 15 \\
\hline CFD & 2000 & 2000 & - \\
\hline TDC & & & 622 \\
\hline
\end{tabular}

(RMS) crystal-to-crystal variation in the measured timing resolution is also shown in Table 1 , and is well under $10 \%$, indicating that the measurements made with several "identical" components are quite reproducible. Subtracting (in quadrature) the $221 \mathrm{ps}$ fwhm reference value, we find that the "extra" contribution to the timing resolution caused by the scintillator crystal (more specifically, the difference between the LSO crystal used in the high-performance setup and those used in the Accel) is 326 ps and 451 ps fwhm for batch $1 \& 2$ respectively.

It is also possible that the average propagation time (i.e., the average time between when the gamma ray interacts and the time that the light pulse exits the scintillator crystal) can vary from crystal to crystal. In order to measure this variation in propagation time, we measure the position of the centroid of the timing distribution for each crystal, compute the RMS variation of these centroid positions, and convert this into a fwhm by multiplying by 2.36 (this assumes a Gaussian distribution). The results, shown in Table 2, are $17 \mathrm{ps}$ and 28 ps fwhm for batch 1 and 2 respectively.

It has previously been shown both theoretically and experimentally that the timing resolution scales inversely as the square root of the initial photoelectron rate (i.e., the number of photoelectrons per ns recorded by the PMT at the leading edge of the PMT output pulse), assuming that the photoelectrons are spaced in time such that their impulse responses overlap [29-32]. This rate is, in turn, proportional to the total light output of the scintillator divided by its decay lifetime (assuming a single exponential decay). If we assume that the LSO decay time is the same for all crystals (previous work has shown this to be a reasonable assumption [23, 33]), the timing resolution will then depend on the square root of the light output.

We therefore test to see whether the timing resolution

Table 2: Component to component variation in the average propagation time of each type of component in the Accel PET camera.

\begin{tabular}{|l|c|c|}
\hline Component & $\begin{array}{c}\text { Propagation Time } \\
\text { Variation (ps fwhm) }\end{array}$ & $\begin{array}{c}\text { Number of } \\
\text { Components }\end{array}$ \\
\hline $\begin{array}{l}\text { Scintillator } \\
\text { (batch 1) }\end{array}$ & 17 & 6 \\
\hline $\begin{array}{l}\text { Scintillator } \\
\text { (batch 2) }\end{array}$ & 28 & 6 \\
\hline $\begin{array}{l}\text { PMT } \\
\text { (Photonis) }\end{array}$ & 484 & 8 \\
\hline $\begin{array}{l}\text { PMT } \\
\text { (Hamamatsu) }\end{array}$ & 274 & 8 \\
\hline CFD & 3278 & 15 \\
\hline
\end{tabular}

Table 3: Dependence of coincidence timing resolution on light output. The errors in the Measured Resolution and Relative Light Output represent the RMS variation over measurements from multiple crystals (six for each batch). These errors are propagated to estimate the errors in the final two columns.

\begin{tabular}{|l|c|c|c|c|}
\hline Component & $\begin{array}{c}\text { Measured } \\
\text { Resol. } \\
\text { (ps fwhm) }\end{array}$ & $\begin{array}{c}\text { Relative } \\
\text { Light } \\
\text { Output }\end{array}$ & $\begin{array}{c}\text { Correction } \\
\text { Factor }\end{array}$ & $\begin{array}{c}\text { Corrected } \\
\text { Resol. } \\
\text { (ps fwhm) }\end{array}$ \\
\hline $\begin{array}{l}\text { Scintillator } \\
\text { (batch 1) }\end{array}$ & $394 \pm 13$ & $1.00 \pm .06$ & $1.00 \pm 0.03$ & $394 \pm 18$ \\
\hline $\begin{array}{l}\text { Scintillator } \\
\text { (batch 2) }\end{array}$ & $502 \pm 17$ & $0.56 \pm .05$ & $0.75 \pm 0.04$ & $376 \pm 24$ \\
\hline
\end{tabular}




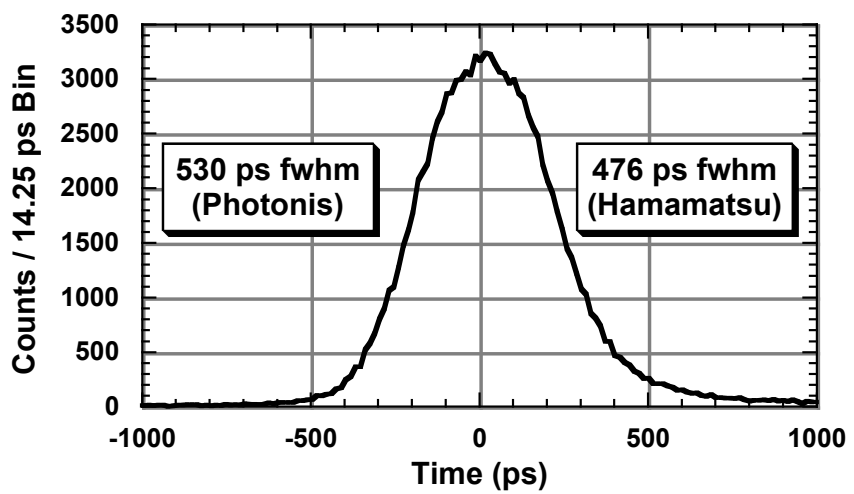

Figure 5: Coincidence timing resolution when a representative Hamamatsu "production" PMT is substituted into the "high-performance" system.

difference between scintillator batches is consistent with differences in the amount of light collected. This "light output" is measured by connecting the output of the PMT with the Accel crystal to a shaper amplifier (Ortec 672 with $1 \mu \mathrm{s}$ shaping time) and obtaining the pulse height spectrum under ${ }^{68} \mathrm{Ge}$ excitation. The center of the $511 \mathrm{keV}$ photopeak is measured and used as an estimate of the relative light output (a crystal from batch 1 is used as a standard). The average light output (i.e., the photopeak position averaged over the crystals in each batch) is computed and shown in Table 3. A correction factor based on the square root of the relative light output is computed and then multiplied by the measured resolution to compute a timing resolution that is "corrected" for the difference in light output. As Table 3 shows, the "corrected" timing resolutions are consistent with each other, suggesting that the difference in LSO light output from crystals in different batches is the cause for their difference in timing resolution.

\section{B. Photomultiplier Tube}

We procured four Accel PMT assemblies from CPS Innovations. Each assembly consists of four $25 \mathrm{~mm}$ diameter PMTs (Photonis 3102FLD1 or Hamamatsu R8619-02) arranged in a $2 \times 2$ matrix and potted in a white reflector material to form a $50 \mathrm{~mm} \times 50 \mathrm{~mm}$ assembly, and is operated at $+1050 \mathrm{~V}$. The "high-performance" $3 \times 8 \times 12 \mathrm{~mm}^{3}$ LSO scintillator crystal is placed on one of the PMTs, and the gain of the PMT is adjusted via a trim pot in the bleeder so that the response to $511 \mathrm{keV}$ excitation (as determined by the photopeak position after shaping with an Ortec 672 amplifier with $1 \mu$ s shaping time) is the same for all PMTs.

The shaper amplifier is disconnected and the PMT is read out with the "high-performance" CFD and TAC/ADC, and the coincidence timing resolution is measured (a typical distribution is shown in Figure 5). This measurement is repeated for each of the 16 individual PMTs in the four assemblies, and the average resolution is $530 \mathrm{ps}$ fwhm for the Photonis PMTs and 476 ps fwhm for the Hamamatsu PMTs. This implies that the "extra" contribution due to the "production" PMT (as opposed to using a "highperformance" PMT) is $482 \mathrm{ps}$ and $422 \mathrm{ps}$ fwhm for the Photonis and Hamamatsu PMTs respectively (221 ps subtracted in quadrature from $530 \mathrm{ps}$ and $476 \mathrm{ps}$ ). The PMT

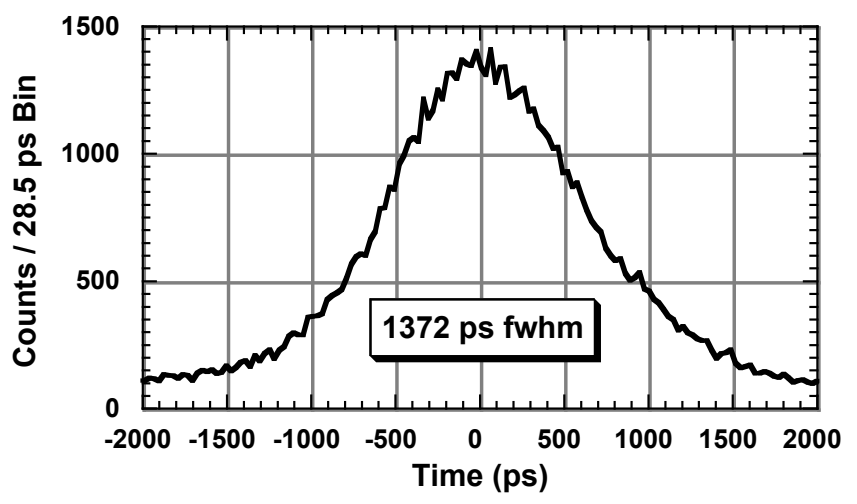

Figure 6: Coincidence timing resolution when a representative "production" CFD is substituted into the "high-performance" system.

to PMT variation in average propagation time is computed as 2.36 times the standard deviation of the centroid of the individual timing distributions, and is found to be $484 \mathrm{ps}$ and $274 \mathrm{ps}$ fwhm for the Photonis and Hamamatsu PMTs respectively. The average timing resolution and the PMT to PMT variation in propagation time are shown in Tables $1 \& 2$ respectively.

\section{Constant Fraction Discriminator}

In the Accel camera, the constant fraction discriminator is part of the CPS Analog ASIC [34-36]. The main components of this ASIC are variable-gain preamplifiers, analog summing circuits, gated integrators, and a non-delay line CFD, and it internally terminates each PMT input with $50 \Omega$. We procured 15 Accel Analog ASICs from CPS Innovations and used them as the CFD in the "high-performance" timing chain (i.e., the system is the $3 \times 8 \times 12 \mathrm{~mm}^{3}$ LSO scintillator crystal, the CPS Analog ASIC, the Ortec 556 TAC, and the National Instruments PXI-7831R 16-bit ADC). Each Analog ASIC is adjusted (gain and delay) to optimize its timing. The average timing resolution with this setup is $1372 \mathrm{ps}$ fwhm (a typical distribution is shown in Figure 6), implying the "extra" contribution to the timing resolution of the "production" CFD of $1354 \mathrm{ps}$ fwhm (221 ps subtracted in quadrature from 1372 ps). These data are also included in Table 1. The CFD to CFD variation in propagation time is again computed as 2.36 times the standard deviation of the centroid of the individual timing distributions. It is found to be $3278 \mathrm{ps}$ fwhm, and is also included in Table 2.

\section{Time to Digital Converter}

In the Accel camera, the time digitization is done with a tapped delay line using $2 \mathrm{~ns}$ taps [37]. Thus, the least significant bit of the production TDC is $2 \mathrm{~ns}$, which defines the timing resolution contribution of this component.

\section{MOdule EFFECTS}

In Section III we measure the "extra" contribution to the timing resolution caused by each individual component in the timing chain: the scintillator crystal, the PMT, the CFD, and the TDC. However, these measurements are made using a single crystal and single PMT (not a block detector module), and it is possible that there is additional degradation because 
of differences between a single crystal and a module. For example, the timing resolution may depend on the position of the individual crystal within the array, or there may be an overall degradation due to reflections off the surfaces and reflector boundaries in the array. In addition, summing the outputs of the four PMTs in a module may result in different timing resolution than with a single PMT. In this section we explore whether there are additional block-related contributions to the timing resolution, or whether the individual contributions measured in Section III are sufficient to describe the performance.

\section{A. Scintillator Crystal Array}

We measure the effects caused by light path variation in the scintillator crystal array in a block detector by attaching a single Accel scintillator crystal (batch 1) to a single, $50 \mathrm{~mm}$ diameter PMT (a fast, 8 stage, experimental PMT from Hamamatsu Photonics) and measuring the timing resolution using the "high-performance" electronics. The measurement is repeated at four positions on the face of the PMT (ranging from the center to the perimeter of the PMT) and averaged, resulting in a mean timing resolution of $355 \pm 37 \mathrm{ps}$ fwhm, where the error is the RMS variation in the 5 measurements. This is consistent with the $326 \mathrm{ps}$ fwhm measured with crystals from this batch on the H-5321 PMT. Next, an Accel scintillator crystal array from CPS Innovations is coupled to the $50 \mathrm{~mm}$ diameter PMT, a $\sim 10 \mathrm{~mm}$ diameter region is excited using electronic collimation, and the timing resolution measured with the "high-performance" electronics. The timing measurement is repeated for 6 more regions (ranging from the array center to the corner) and the measurements averaged, giving a timing resolution for the scintillator array of $577 \pm 24 \mathrm{ps}$ fwhm, where again the error represents the RMS variation in the 7 measurements.

It is tempting to attribute the difference in timing resolution between the single crystal and the module to light propagation in the module, etc. However, the light output in this crystal array (averaged over all 7 positions) is a factor of 3.0 smaller than the batch 1 single crystal. The light output is quite uniform in the scintillator array-in this block the crystal to crystal variation in the position of the $511 \mathrm{keV}$ photopeak is only 6\% RMS. As the timing resolution is theoretically expected to scale inversely with the square root of the light collected, we use the measured time resolution and light output to compute a "corrected" timing resolution for the crystal array of $333 \pm 16 \mathrm{ps}$ fwhm. As this is consistent with the $355 \pm 37$ ps measured the single crystal, we conclude that the difference between the timing of an individual scintillator crystal and a detector module is consistent with the difference in the amount of light collected.

\section{B. PMT Assembly}

We also measure the effect caused by the scintillation light being shared by four PMTs. First, we attach a single Accel scintillator crystal from batch 1 to one of the Hamamatsu "production" PMTs in a 4 PMT assembly and measure the timing resolution using the "high-performance" electronics.
Table 4: Dependence of coincidence timing resolution on light output. The errors in the Measured Resolution and Relative Light Output represent the RMS variation over measurements at multiple positions (seven for each array). These errors are propagated to estimate the errors in the final two columns.

\begin{tabular}{|l|c|c|c|c|}
\hline Component & $\begin{array}{c}\text { Measured } \\
\text { Resol. } \\
\text { (ps fwhm) }\end{array}$ & $\begin{array}{c}\text { Relative } \\
\text { Light } \\
\text { Output }\end{array}$ & $\begin{array}{c}\text { Correction } \\
\text { Factor }\end{array}$ & $\begin{array}{c}\text { Corrected } \\
\text { Resol. } \\
\text { (ps fwhm) }\end{array}$ \\
\hline $\begin{array}{l}\text { Single Xtal } \\
\text { (batch 1) }\end{array}$ & 577 & 1.08 & 1.04 & 600 \\
\hline Array 1 & $1292 \pm 84$ & $0.23 \pm 0.01$ & $0.48 \pm 0.01$ & $625 \pm 44$ \\
\hline Array 2 & $789 \pm 65$ & $0.49 \pm 0.03$ & $0.70 \pm 0.02$ & $552 \pm 47$ \\
\hline Array 3 & $916 \pm 78$ & $0.60 \pm 0.01$ & $0.77 \pm 0.01$ & $707 \pm 59$ \\
\hline Array 4 & $996 \pm 172$ & $0.60 \pm 0.02$ & $0.78 \pm 0.01$ & $774 \pm 131$ \\
\hline
\end{tabular}

An analog sum of the 4 PMT outputs is then formed by attaching each PMT output to the CFD input through a $21 \Omega$ resistor. The timing measurement is repeated and the timing resolution (for a single crystal attached to a single PMT) is found to be the same when the PMT is read out independently and when summed with three other PMTs. The crystal is moved to each of the other three PMTs in the assembly and similar results obtained. We conclude that summing the PMT outputs in this manner does not affect the timing resolution of the individual PMTs.

We then couple a scintillator array to the PMT assembly, read out the summed PMT outputs with the "highperformance" electronics, excite a $\sim 10 \mathrm{~mm}$ diameter region in the array using electronic collimation, and measure the coincidence timing resolution. The measurement is averaged over seven positions within the array (arranged in a V-shaped pattern going from one corner of the module to the center and then back to an adjacent corner) and is performed for all four scintillator arrays, and the results shown in Table 4. The small size of the errors in Table 4 indicates that the performance (both timing resolution and light output) is very uniform within a single module. Significant differences between arrays and between the single crystal and the arrays are observed, but when scaled by the square root of the light output (also shown in Table 4), the results are consistent.

We also explore the effect of energy threshold on the timing resolution for these arrays by accepting only events whose energy deposit is within $\pm 20 \%$ of $511 \mathrm{keV}$ as opposed to the default value of $>50 \mathrm{keV}$. This does not significantly affect the timing resolution - the average improvement over the timing resolution presented in Table 4 is only $30 \mathrm{ps,}$ which is within statistical error. A possible explanation for this insensitivity to threshold is that most annihilation photons deposit a large fraction of their energy in the detector block and thus lie in the "photopeak" window. If few events lie outside the photopeak window, including these events (by using a low energy threshold) will not significantly affect the measured timing resolution.

\section{DISCUSSION}

The data presented in Table 1 represent the "extra" contributions to the timing resolution of the four major components in the timing chain of the Accel PET camera: the 
scintillator crystal, the PMT, the CFD, and the TDC. It is clear that the contributions from the electronics (the CFD and the TDC) dominate, and are the main reason that the timing resolution of the Accel is $2-3 \mathrm{~ns}$ fwhm. This is not surprising, as these electronics were originally designed for BGO-based PET cameras for which such timing resolution is appropriate. The TDC has a 2 ns least count, and within the ASIC the amplifier bandwidth is a few hundred megahertz and the CFD uses a filter (that attenuates high frequency components of the signal) instead of a delay line. The recently developed "Pico-Timing" electronics option $[38,39]$ is likely to provide significantly better timing resolution.

The data also suggest that these four components (scintillator, PMT, CFD, and TDC), the component-tocomponent propagation time differences (Table 2), and the total amount of light seen by the PMTs are the only factors that contribute significantly to the timing resolution. In particular, measurements made on single crystals and single PMTs are consistent with (and thus can substitute for) measurements of block detector modules that contain scintillator crystal arrays and multiple PMTs, provided that the differences in the light collection are accounted for.

Finally, these data can be used to predict the achievable timing resolution of an LSO-based PET camera. The timing resolution for a module can be accurately estimated by the quadrature sum of the "high-performance" LSO / PMT combination (162 ps) and the relevant "extra" contributions, and then scaling by the reciprocal of square root of the relative light output. The relevant "extra" contributions can be due to the choice of the single scintillator crystal, PMT, or CFD used, as well as the component-to-component variations in propagation time in the scintillator crystal and PMTs.

The data in Table 1 indicate that the electronics is currently the largest bottleneck in the Accel. If we assume that commercial PET camera electronics can be made to perform as well as existing NIM electronics, then the "extra" contributions for the CFD and TDC will drop to zero. The remaining factors due to individual components (Table 1) are the scintillator crystal ( $325 \mathrm{ps})$ and the PMT ( $450 \mathrm{ps})$. We must also include contributions for the component-tocomponent propagation delay variation in the various components (Table 2). The contribution from the scintillator crystal is negligible ( $20 \mathrm{ps}$ fwhm), but the PMT to PMT variation in propagation delay is a non-trivial ( $\sim 275 \mathrm{ps})$ factor. The factor due to CFD to CFD variation in propagation time ( $3278 \mathrm{ps}$ ) can be ignored, as all the signals in a module go through a single CFD and thus are all skewed in time by the same amount. This module dependent time skew is eliminated via calibration. The magnitude of this CFD propagation time variation is surprisingly large, and may be why other PET cameras using the same ASIC require relatively large time skew corrections for each module [40]. Finally, the effect of light output (a multiplicative factor between $1 / 1.0$ and $1 / 0.5$, Table 4) must be included.

The PMT to PMT propagation time variation can probably be reduced or removed by selecting PMTs with similar propagation time or using individual delay cables to time-
Table 5: Present and potential factors that contribute to the timing resolution of the Accel PET camera. The Single Module Timing is equal to the Light Output factor times the quadrature sum of the individual factors. The Coincidence Timing is the Single Module Timing times the square root of two, then added in quadrature with the TDC factor.

\begin{tabular}{|l|c|c|}
\hline Component & $\begin{array}{c}\text { Present Timing } \\
\text { Factor } \\
\text { (ps fwhm) }\end{array}$ & $\begin{array}{c}\text { Potential } \\
\text { Timing Factor } \\
\text { (ps fwhm) }\end{array}$ \\
\hline Baseline & 162 & 162 \\
\hline Crystal Geometry & 325 & 250 \\
\hline Crystal Variation & 20 & 20 \\
\hline PMT Type & 450 & 0 \\
\hline PMT Variation & 275 & 0 \\
\hline CFD & 1372 & 0 \\
\hline Quadrature Sum of Above & 1514 & 299 \\
\hline $\begin{array}{l}\text { Light Output } \\
\text { (multiplicative factor) }\end{array}$ & $1.25-2.0$ & $1.25-1.42$ \\
\hline Single Module Timing & $\mathbf{1 8 9 3 - 3 0 2 8}$ & $\mathbf{3 7 4 - 4 2 5}$ \\
\hline TDC & 2000 & 0 \\
\hline Coincidence Timing & $\mathbf{3 3 4 2 - 4 7 2 7}$ & $\mathbf{5 2 8}-\mathbf{6 0 0}$ \\
\hline
\end{tabular}

align the four PMTs within each module. The factor due to the PMT type can probably be reduced significantly by using PMTs with a smaller number of stages (e.g., 8), such as the experimental $50 \mathrm{~mm}$ PMT used in Section IV.A. The cost, stability, and energy resolution of these PMTs are very appropriate for commercial PET cameras, thus the "extra" factors due to the PMTs (both PMT type and propagation time variation) can probably be reduced to zero. It is unlikely that the factor due to the scintillator crystal can be significantly reduced, as it probably is due to the long, thin geometry that is necessary for PET. However, previous work has indicated that a mechanically polished surface has $\sim 20 \%$ better timing resolution than a chemically polished surface [16], so partial reduction of this factor to $\sim 250 \mathrm{ps}$ fwhm may be possible. This is the only "extra" factor or component to component propagation time variation that is unlikely to be reduced to zero in a commercial LSO-based PET camera.

The final factor is the scale factor based on how the amount light collected in a detector compares to that of the best single crystal. Some of the difference may be due to boule-to-boule variations in the intrinsic LSO light output and some may be due to the process of assembling the individual crystals to form a module. Both of these involve details in the manufacturing process, and so neither their cause, magnitude, nor cure is known to us. Thus, we estimate that the value of the correction factor will be between $1 / 0.7$ (which is the mean value of the four modules that we possess) and 1/0.8 (which is the best value of the four modules that we possess). We know that this multiplicative factor will not be less than 1.0 (which would imply better light output in a module than in an individually coupled crystal) nor is it likely to be greater than 2.0 (which implies a factor of four lower light output in a module). As the ultimate timing resolution depends critically on the light output, care must be taken to ensure that the highest possible light output is obtained.

Table 5 summarizes the present measured values and the values that we believe to be possible, given the assumptions in the previous two paragraphs (162 ps baseline, $250 \mathrm{ps}$ "extra" factor from the scintillator geometry, and a factor of 
$1 / 0.8$ for the relative light output). The best possible timing resolution expected for a single LSO-based block detector module is $374 \mathrm{ps}$ fwhm, implying a coincidence timing resolution $528 \mathrm{ps}$ fwhm. If the more conservative relative light output factor of $1 / 0.7$ is used, we predict a single LSObased block detector module timing resolution of $425 \mathrm{ps}$ fwhm and a coincidence timing resolution $600 \mathrm{ps}$ fwhm. This would be accomplished with high performance (but cost effective) electronics and PMTs and would require selecting PMTs that have similar propagation times. It also assumes polished scintillator crystals and scintillator arrays whose light output is between $49 \%$ and $64 \%$ that of a single LSO crystal directly coupled to a PMT.

There is a caveat regarding energy threshold. The exact value of the timing resolution usually depends on the energy threshold, as events with lower energy deposit (and thus lower initial photoelectron rate) will have worse timing resolution than events with a larger energy deposit. This fact has little impact on the majority of this paper, which is concerned with relative contributions to timing resolution (i.e., which factors dominate). However, absolute numbers are preferable when predicting the performance of a complete PET system. The resolution predicted in this section (and listed in Table 5) is based on data obtained with a low energy threshold $(<50 \mathrm{keV})$, so the absolute value of the timing resolution may improve if a higher energy threshold is used. However, the measurements presented at the end of Section III suggest that such an improvement would be modest, implying that the coincidence timing resolution predicted in this Section (528-600 ps fwhm) is likely to be representative of a PET camera (that only accepts events that are in the photopeak window).

\section{CONCLUSIONS}

We explore the contributions to the coincidence timing resolution in the CPS Accel, a commercial LSO-based PET camera. The main contributions are due to the present electronics, which were originally designed for a BGO-based PET camera that would not benefit from improved timing electronics. Additional smaller factors result from the shape of the scintillator crystal, the choice of PMT, and component to component variations between PMTs. Measurements made on individual crystals and PMTs accurately predict the performance of a block detector module, indicating that forming a block detector (i.e., sharing light in the scintillator array and between PMTs) does not significantly affect the timing resolution. However, the amount of light collected by the PMT is important, as the timing resolution scales inversely with the square root of the light collected. Based on these measurements we believe that with current technology, the limit for the coincidence timing resolution in a practical, commercial, LSO-based PET camera is 528 ps fwhm.

\section{ACKNOWLEDGMENTS}

We thank Dr. Michael Casey and Dr. Bernard Bendriem of CPS Innovations, and Mr. Sam Hales of Hamamatsu
Photonics for supplying invaluable components and information. This work was supported in part by the Director, Office of Science, Office of Biological and Environmental Research, Medical Science Division of the U.S. Department of Energy under Contract No. DE-AC02-05CH11231, and in part by the National Institutes of Health, National Institute of Biomedical Imaging and Bioengineering under grant No. R33-EB001928. Reference to a company or product name does not imply approval or recommendation by the University of California or the U.S. Department of Energy to the exclusion of others that may be suitable.

\section{REFERENCES}

[1] M. M. Ter-Pogossian, D. C. Ficke, M. Yamamoto and J. T. Hood. Design characteristics and preliminary testing of Super-PETT I, a positron emission tomograph utilizing photon time-of-flight information (TOF PET). Proceedings of The Workshop on Time of Flight Tomography, pp. 37-41, (St. Louis, MO, May 1982.

[2] M. M. Ter-Pogossian, D. C. Ficke, M. Yamamoto and J. T. Hood, "Super PETTI: A positron emission tomograph utilizing photon timeof-flight information," IEEE Trans. Med. Img., vol. MI-1, pp. 179-187, Nov. 1982.

[3] N. A. Mullani, W. H. Wong, R. K. Hartz, K. Yerian, E. A. Philippe, et al. Design of TOFPET: a high resolution time-of-flight positron camera. Proceedings of The Workshop on Time of Flight Tomography, pp. 31-36, (St. Louis, MO, May 1982.

[4] R. Gariod, R. Allemand, E. Cormoreche, M. Laval and M. Moszynski. The LETI positron tomograph architecture and time of flight improvements. Proceedings of The Workshop on Time of Flight Tomography, pp. 25-29, (St. Louis, MO, May 1982.

[5] W. H. Wong, N. A. Mullani, E. A. Phillipe, R. Hartz and K. L. Gould, "Image improvement and design optimization of the time-of-flight PET," J. Nucl. Med., vol. 24, pp. 52-60, Jan. 1983.

[6] T. K. Lewellen, A. N. Bice, R. L. Harrison, M. D. Pencke and J. M. Link, "Performance measurements of the SP3000/UW time-of-flight positron emission tomograph," IEEE Trans. Nucl. Sci., vol. NS-35, pp. 665-669, Feb. 1988.

[7] B. Mazoyer, R. Trebossen, C. Schoukroun, B. Verrey, A. Syrota, et al., "Physical characteristics of TTV03, a new high spatial resolution timeof-flight positron tomograph," IEEE Trans. Nucl. Sci., vol. NS-37, pp. 778-782, Apr. 1990

[8] M. M. Ter-Pogossian, D. C. Ficke, D. E. Beecher, G. R. Hoffmann and S. R. Bergmann, "The Super PET 3000-E - a PET scanner designed for high count rate cardiac applications," J. Comput. Assist. Tomogr., vol. 18, pp. 661-669, July 1994.

[9] T. K. Lewellen, "Time-of-Flight PET," Semin. Nucl. Med., vol. 28, pp. 268-275, July 1998.

[10] W. W. Moses and K. S. Shah, "Potential for $\mathrm{RbGd}_{2} \mathrm{Br}_{7}: \mathrm{Ce}, \mathrm{LaBr}_{3}: \mathrm{Ce}$, $\mathrm{LaBr}_{3}: \mathrm{Ce}$, and $\mathrm{LuI}_{3}: \mathrm{Ce}$ in nuclear medical imaging," Nucl. Instr. Meth., vol. A-537, pp. 317-320, Jan. 2005.

[11] K. S. Shah, L. Cirignano, R. Grazioso, M. Klugerman, P. R. Bennett, et al., " $\mathrm{RbGd}_{2} \mathrm{Br}_{7}$ : $\mathrm{Ce}$ scintillators for gamma ray and thermal neutron detection," IEEE Trans. Nucl. Sci., vol. NS-49, pp. 1655-1660, Aug. 2002.

[12] K. S. Shah, Y. Glodo, M. Klugerman, L. Cirignano, W. W. Moses, et al., " $\mathrm{LaCl}_{3}: \mathrm{Ce}$ scintillators for gamma ray detection," Nucl. Instr. Meth., vol. A-505, pp. 76-81, June 2003.

[13] K. S. Shah, J. Glodo, M. Klugerman, W. W. Moses, S. E. Derenzo, et al., " $\mathrm{LaBr}_{3}$ :Ce scintillators for gamma ray spectroscopy," IEEE Trans. Nucl. Sci., vol. NS-50, pp. 2410-2413, Dec. 2003.

[14] K. S. Shah, J. Glodo, W. Higgins, E. V. D. van Loef, W. W. Moses, et al. $\mathrm{CeBr}_{3}$ scintillators for gamma ray spectroscopy. Proceedings of The IEEE 2004 Nuclear Science Symposium, pp. R4-1, (Edited by A. Siebert), Rome, Italy, 2004.

[15] K. S. Shah, J. Glodo, M. Klugerman, W. Higgins, T. Gupta, et al., "LuI $\mathrm{I}_{3}$ : $\mathrm{Ce}-\mathrm{a}$ new scintillator for gamma ray spectroscopy," IEEE Trans. Nucl. Sci, vol. NS-51, pp. 2302-2305, Oct. 2004.

[16] W. W. Moses and S. E. Derenzo, "Prospects for time-of-flight PET using LSO scintillator," IEEE Trans. Nucl Sci., vol. NS-46, pp. 474478, June 1999. 
[17] W. W. Moses, “Time of flight in PET revisited," IEEE Trans. Nucl. Sci., vol. NS-50, pp. 1325-1330, Oct. 2003.

[18] S. Surti, J. S. Karp, and G. Muehllehner. Image quality assessment of $\mathrm{LaBr}_{3}$ based 3D PET scanners. Proceedings of The IEEE 2003 Nuclear Science Symposium and Medical Imaging Conference, pp. M3-148, (Edited by S. D. Metzler), Portland, OR, 2003.

[19] A. Kuhn, S. Surti, J. S. Karp, P. S. Raby, K. S. Shah, et al., "Design of a lanthanum bromide detector for time-of-flight PET," IEEE Trans. Nucl. Sci., vol. NS-51, pp. 2550-2557, Oct. 2004.

[20] A. Kuhn, S. Surti, J. S. Karp, G. Muehllehner, F. M. Newcomer, et al. Performance assessment of pixellated $\mathrm{LaBr}_{3}$ detector modules for TOF PET. Proceedings of The IEEE 2004 Nuclear Science Symposium, pp. M9-59, (Edited by A. Siebert), Rome, Italy, 2004.

[21] S. Surti, J. S. Karp, L. M. Popescu, M. E. Daube-Witherspoon and M. Werner. Investigation of image quality and NEC in a TOF-capable PET scanner. Proceedings of The IEEE 2004 Nuclear Science Symposium, pp. M10-276, (Edited by A. Siebert), Rome, Italy, 2004.

[22] C. L. Melcher and J. S. Schweitzer, "Cerium-doped lutetium orthosilicate: a fast, efficient new scintillator," IEEE Trans. Nucl. Sci., vol. NS-39, pp. 502-505, Aug. 1992.

[23] C. L. Melcher, M. A. Spurrier, M. J. Schmand, L. A. Eriksson and R. Nutt, "Advances in the scintillation performance of LSO:Ce single crystals," IEEE Trans. Nucl. Sci., vol. NS-50, pp. 762-766, Aug. 2003.

[24] M. Moszynski, T. Ludziejewski, D. Wolski, W. Klamra and V. V. Avdejcikov, "Timing properties of GSO, LSO and other Ce doped scintillators," Nucl. Instr. Meth., vol. A 372, pp. 51-58, Mar. 1996.

[25] M. Schmand, L. Eriksson, M. E. Casey, M. S. Andreaco, C. Melcher, et al., "Performance results of a new DOI detector block for a High Resolution PET-LSO Research Tomograph HRRT," IEEE Trans. Nucl. Sci., vol. NS-45, pp. 3000-3006, Dec. 1998.

[26] M. Schmand, K. Wienhard, M. E. Casey, L. Eriksson, W. F. Jones, et al. Performance evaluation of a new LSO high resolution research tomograph-HRRT. Proceedings of The IEEE 1999 Nuclear Science Symposium and Medical Imaging Conference, pp. 1067-1071, vol. 2, paper M04-002, (Edited by J. A. Seibert), Seattle, WA, 1999.

[27] K. Wienhard, M. Schmand, M. E. Casey, K. Baker, J. Bao, et al., "The ECAT HRRT: performance and first clinical application of the new high resolution research tomograph," IEEE Trans. Nucl. Sci., vol. NS49, pp. 104-110, Feb. 2002.

[28] T. J. Spinks and P. M. Bloomfield. A comparison of count rate performance for ${ }^{15} \mathrm{O}$-water blood flow studies in the CTI HR+ and Accel tomographs in 3D mode. Proceedings of The IEEE 2002 Nuclear Science Symposium and Medical Imaging Conference, pp. M10-59, (Edited by S. Metzler), Norfolk, VA, 2002.

[29] R. F. Post and L. I. Schiff, "Statistical limitations on the resolving time of a scintillation counter," Phys. Rev., vol. 80, pp. 1113-1120, Dec. 1950.

[30] L. G. Hyman, R. M. Schwarcz, and R. A. Schluter, "Study of high speed photomultiplier tube systems," Rev. Sci. Instr., vol. 35, pp. 393406, Mar. 1964.

[31] L. G. Hyman, "Time resolution of photomultiplier tube systems," Rev. Sci. Instr., vol. 36, pp. 193-196, Feb. 1965.

[32] C. C. Lo and B. Leskovar, "A measuring system for studying the timeresolution capabilities of fast photomultipliers," IEEE Trans. Nucl. Sci., vol. NS-24, pp. 93-105, Feb. 1977.

[33] C. L. Melcher, M. Schmand, M. Eriksson, L. Eriksson, M. Casey, et al., "Scintillation properties of LSO:Ce boules," Nuclear Science, IEEE Transactions on, vol. 47, pp. 965-968, June 2000.

[34] D. M. Binkley, M. L. Simpson, and J. M. Rochelle, "A monolithic, $2 \mu \mathrm{m}$ CMOS constant-fraction discriminator for moderate time resolution systems," IEEE Trans. Nucl. Sci., vol. 38, pp. 1754-1759, Dec. 1991.

[35] D. M. Binkley, "Performance of non-delay-line constant-fraction discriminator timing circuits," IEEE Trans. Nucl. Sci., vol. 41, pp. 1169-1175, Aug. 1994.

[36] D. M. Binkley, B. S. Puckett, B. K. Swann, J. A. Rochelle, M. S. Musrock, et al., "A $10-\mathrm{mc} / \mathrm{s}, \quad 0.5-\mu \mathrm{m}$ CMOS constant-fraction discriminator having built-in pulse tail cancellation," IEEE Trans. Nucl. Sci., vol. NS-49, pp. 1130-1140, June 2002.

[37] J. W. Young, J. C. Moyers, and M. Lenox, "FPGA based front end electronics for a high resolution PET scanner," IEEE Trans. Nucl. Sci., vol. NS-47, pp. 1676-1680, Aug. 2000.

[38] B. K. Swann, J. M. Rochelle, D. M. Binkley, B. S. Puckett, B. J. Blalock, et al., "A custom mixed-signal CMOS integrated circuit for high performance PET tomograph front-end applications," IEEE Trans. Nucl. Sci., vol. NS-50, pp. 909-914, Aug. 2003.

[39] M. S. Musrock, J. W. Young, J. C. Moyers, J. E. Breeding, M. E. Casey, et al., "Performance characteristics of a new generation of processing circuits for PET applications," IEEE Trans. Nucl. Sci., vol. NS-50, pp. 974-978, Aug. 2003.

[40] C. J. Thompson and A. Goertzen, "Measurements on the timing stability of the MicroPET R4 animal PET scanner," IEEE Trans. Med. Img., (accepted for publication), 2005. 